\title{
Prognostic significance of the lung scintigraphy scan result and corresponding chest X-ray in patients with suspected pulmonary embolism
}

\author{
${ }^{1}$ AS Al-Adhami, ${ }^{2} \mathrm{AJ}$ Simpson, ${ }^{3} \mathrm{JH}$ Reid, ${ }^{4} \mathrm{M}$ MacDougall, ${ }^{5} \mathrm{JT}$ Murchison \\ ${ }^{1}$ Specialist Registrar, West of Scotland Training Scheme; ${ }^{2}$ Senior Clinical Lecturer in Respiratory Medicine, University of Edinburgh; ${ }^{3}$ Consultant \\ Radiologist, Department of Radiology, Borders General Hospital, Melrose; ${ }^{4}$ Medical Statistician and Researcher in Education, Division of Community \\ Health Sciences, University of Edinburgh Medical School; ${ }^{5}$ Consultant Radiologist, Department of Radiology, Royal Infirmary of Edinburgh
}

\section{ABSTRACT}

Aim: To determine whether the survival of patients with suspected acute pulmonary embolism (PE) relates to radiological probability of acute PE assessed using lung scintigraphy scans (LSS).

Methods: Lung scintigraphy scan results from a venous thromboembolism database were categorised as high, indeterminate or low probability using the modified PIOPED criteria and corresponding chest X-rays (CXRs) as normal or abnormal. Mortality data on these cases were obtained from the General Register Office for Scotland, and survival was analysed using the Kaplan-Meier method.

Results: Of the I,8I8 LSS analysed, 94I (5I.8\%) were normal, 532 (29.3\%) indeterminate and 345 (19.0\%) high probability. After an adjustment for age and gender, no significant survival difference was found between patients with normal and high probability LSS $(p=0.182)$. However, patients with indeterminate LSS had significantly lower survival than patients in the other groups. This difference persisted after adjustment for CXR result.

Conclusions: Indeterminate LSS results are associated with a poor prognosis. Careful follow-up of patients with inderminate LSS would appear to be justified.

KEYWORDS Lung scintigraphy scan, outcome, pulmonary embolism, survival, ventilation perfusion

DECLARATION OF INTERESTS AJ Simpson is the Editor of The Journal of the Royal College of Physicians of Edinburgh. This article was submitted to, and handled exclusively by, the JRCPE's Clinical Editor and has been peer-reviewed in line with usual JRCPE procedures.

\section{INTRODUCTION}

Although most centres now employ computed tomography pulmonary angiography (CTPA) as the firstline investigation for clinically suspected pulmonary embolism (PE), ${ }^{1-2}$ lung scintigraphy scans (LSS) remain a useful imaging modality ${ }^{3}$ which is still widely used. ${ }^{4-7}$ The PIOPED study suggested that high probability LSS are reliable in confirming PE (specificity $97 \%$ ) and normal scans can reliably exclude PE (sensitivity 98\%). ${ }^{5}$ However, almost two-thirds of scans in the PIOPED study were of intermediate or low probability and associated with a $25-40 \%$ risk of PE. All LSS can be categorised as either high probability of $\mathrm{PE}$, indeterminate probability of $\mathrm{PE}$, or normal. The aim of this study was to test the hypothesis that survival would be inversely related to the radiological probability of PE as assessed by LSS performed at the time of presentation with clinically suspected PE.

\section{METHODS}

Results from 2,092 LSS performed at a single teaching hospital between April 1994 and August 2000 were prospectively entered onto a database and retrospectively
Correspondence to JT Murchison, Department of Clinical Radiology, Royal Infirmary of Edinburgh, Edinburgh EHI6 4SU, UK

tel. +44 (0) 13। 2423775

e-mail

john.murchison@luht.scot.nhs.uk reviewed. During this period LSS were the first-line diagnostic procedure used at this hospital for investigation of suspected PE. Computed tomography pulmonary angiography was introduced towards the end of this period but was initially used to problem-solve non-diagnostic LSS. ${ }^{3}$ Radiologists reported LSS using modified PIOPED criteria throughout this time period. ${ }^{5}$ Scan results were recorded on the database at the time of scanning as normal, indeterminate or high probability. The indeterminate group combined intermediate and low probability categories described by the PIOPED investigators. Radiologists also recorded whether the corresponding chest X-ray (CXR) was normal or abnormal. All CXRs were performed within 24 hours prior to the LSS, where possible using a postero-anterior projection. An Ohio Nuclear Sigma 410 gamma camera was used for scintigraphy, with $80 \mathrm{MBq}$ of technetium-labelled albumin macro-aggregates administered intravenously for perfusion imaging and inhalation of $80 \mathrm{MBq}$ of Xenon-133 or Xenon-127 for ventilation studies when required.

Mortality data were obtained, courtesy of Mr I Brown, from the General Register Office for Scotland (GROS), which registers all deaths in the country. If four personal 


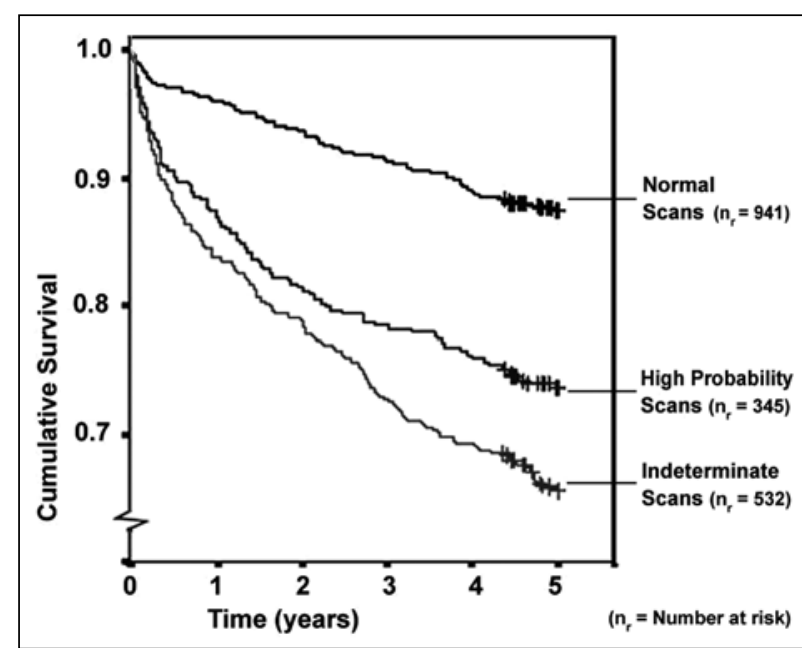

FIGURE I Kaplan-Meier survival curves in patients with normal, indeterminate or high probability scintigraphy scans $(p<0.00$ l overall, $p=0.017$ for the comparison between patients with indeterminate and high probability scans, $p<0.0005$ for the comparisons between patients with normal scans and either of the other two scan groups; log-rank test).

identifiers (surname, date of birth, gender and postcode) matched GROS data, the patient was considered deceased and the date of death recorded. If three of four identifiers matched, other identifiers were manually checked to confirm or refute matching. If no match was found, the patient was considered alive.

Survival time was defined as that between the date of the index LSS and date of death. Surviving patients were censored at five years after the LSS or at the date of attempted matching at GROS if five years had not elapsed (this applied for 67 patients - minimum followup 4.36 years). Survival was analysed using the KaplanMeier method on the Statistical Package for Social Sciences (SPSS). Statistical significance was considered to be at the level of $p<0.05$. Cox regression analysis was used to assess the relative independent contributions of each factor (age, gender, LSS result and CXR result) to survival.

The likelihood ratios (LR) backward stepwise method was used to rank the individual factors. The rule assumed here was a special instance of the Akaike Information Criterion, that the higher the increase in the -2 log likelihood statistic on removal of any one factor from the full model, the more important the factor is in predicting patient outcome. ${ }^{8}$

\section{RESULTS}

Data were excluded if the LSS was an individual's second or subsequent scan during the study period $(n=129)$ or if no Scottish postcode was found $(n=145)$. Therefore I,8I8 LSS remained in the final analysis, of which 94I (5I.8\%) were normal, 532 (29.3\%) indeterminate and 345 (19.0\%) high probability. Normal scans predicted for significantly greater survival rates than high probability
TABLE I Results of Cox regression analysis, adjusting for age and sex

\begin{tabular}{|l|l|l|l|l|}
\hline & p-value & \multirow{2}{*}{$\begin{array}{l}\text { Hazard } \\
\text { ratio }\end{array}$} & \multicolumn{2}{|l|}{$\begin{array}{l}\text { 95\% confidence } \\
\text { interval }\end{array}$} \\
\cline { 4 - 5 } & & & Lower & Upper \\
\hline $\begin{array}{l}\text { Indeterminate } \\
\text { vs normal LSS }\end{array}$ & 0.000 & 1.826 & 1.441 & 2.314 \\
\hline $\begin{array}{l}\text { High probability } \\
\text { vs normal LSS }\end{array}$ & 0.182 & 1.210 & 0.915 & 1.601 \\
\hline $\begin{array}{l}\text { High probability } \\
\text { vs indeterminate } \\
\text { LSS }\end{array}$ & 0.001 & 0.663 & 0.515 & 0.852 \\
\hline $\begin{array}{l}\text { Sex } \\
\text { (male vs female) }\end{array}$ & 0.045 & 1.226 & 1.004 & 1.496 \\
\hline $\begin{array}{l}\text { Age (diffence per } \\
\text { year increase) }\end{array}$ & 0.000 & 1.069 & 1.060 & 1.078 \\
\hline
\end{tabular}

TABLE 2 Results of Cox regression analysis, with adjustment for chest $X$-ray result, age and sex

\begin{tabular}{|l|l|l|l|l|}
\hline & p-value & $\begin{array}{l}\text { Hazard } \\
\text { ratio }\end{array}$ & \multicolumn{2}{|l|}{$\begin{array}{l}\text { 95\% confidence } \\
\text { interval }\end{array}$} \\
\cline { 3 - 5 } & & & Lower & Upper \\
\hline $\begin{array}{l}\text { Indeterminate vs } \\
\text { normal LSS }\end{array}$ & 0.001 & 1.636 & 1.237 & 2.166 \\
\hline $\begin{array}{l}\text { High probability } \\
\text { vs normal LSS }\end{array}$ & 0.332 & 1.174 & 0.849 & 1.622 \\
\hline $\begin{array}{l}\text { High probability } \\
\text { vs indeterminate } \\
\text { LSS }\end{array}$ & 0.024 & 0.717 & 0.538 & 0.956 \\
\hline $\begin{array}{l}\text { Chest X-ray } \\
\text { (abnormal vs } \\
\text { normal) }\end{array}$ & 0.000 & 1.818 & 1.380 & 2.395 \\
\hline $\begin{array}{l}\text { Age (difference } \\
\text { per year increase) }\end{array}$ & 0.000 & 1.065 & 1.055 & 1.074 \\
\hline $\begin{array}{l}\text { Sex (male vs } \\
\text { female) }\end{array}$ & 0.080 & 1.220 & 0.976 & 1.524 \\
\hline
\end{tabular}

and indeterminate scans $(\mathrm{p}<0.00 \mathrm{I})$, with indeterminate scans predicting significantly lower survival rates than high probability scans ( $p=0.017$; log-rank test) (Figure I). The percentage of males in the normal, indeterminate and high probability groups were $42.2 \%, 45.1 \%$ and $45.8 \%$ respectively. The mean ages were 48.9 years, $6 \mathrm{I}$. I years and 62.0 years for the normal, indeterminate and high probability groups respectively.

After adjustment for influences of age and gender (using Cox regression analysis), no significant difference in survival was seen between patients with normal and high probability LSS $(p=0.182)$. However, the difference in survival between patients with indeterminate and high probability LSS was significant $(p=0.00 \mathrm{I})$, with the indeterminate scan predicting a lower survival (Table I). Patients with indeterminate scans also had significantly reduced survival when compared with patients who had normal scans (Table I). 
TABLE 3 The increase in the -2 log likelihood statistic on removal of any of the factors - and the corresponding $\mathrm{p}$-value - from the LR backward stepwise regression. This shows that the appropriate importance ranking for the independent contribution of each factor to the regression model is age $>$ CXR result $>$ LSS result $>$ sex

\begin{tabular}{|l|l|l|l|}
\hline \multicolumn{2}{|l|}{ Term removed } & $\begin{array}{l}\mathbf{- 2} \text { log likelihood } \\
\text { increase }\end{array}$ & p-value \\
\hline Step I & Age & 224.698 & 0.000 \\
\hline & Sex & 3.049 & 0.081 \\
\hline & LSS result & 13.262 & 0.001 \\
\hline & CXR result & 19.398 & 0.000 \\
\hline
\end{tabular}

Chest X-ray data corresponding to I,406 scans (77.3\%) were available. A total of 733 CXRs (52.1\%) were considered normal. The corresponding CXR was abnormal in $26 \%, 78 \%$ and $58 \%$ of patients with a normal, indeterminate and high probability scan respectively. When including the CXR result as an additional variable in the Cox regression analysis, the difference in survival between patients with normal and high probability LSS remained non-significant $(p=0.332)$. However, there was still a statistically significant difference in survival when comparing patients with indeterminate scans and patients in either of the other two groups (Table 2). When the survival analysis in Figure I was repeated using only patients with a normal CXR, no significant difference was found between the (relatively small) subgroups with indeterminate and high probability LSS.

Using the LR backward stepwise method (Table 3), the relative importance of the CXR result on the overall survival was found to be greater than that of the LSS result.

\section{DISCUSSION}

Acute PE occurs most commonly in patients over the age of 60 and has been associated with all-cause mortality rates of around $15 \%$ at three months. ${ }^{9}$ It is suspected clinically far more often than it is confirmed radiologically. A study by Hvitfeldt Poulsen et al. showed that among patients with suspected PE the one-year all-cause mortality for patients with PE confirmed by imaging was not significantly different from that in patients for whom PE was refuted by imaging ( $18 \%$ and $15 \%$ respectively). ${ }^{10}$ A separate study found a six-month all-cause mortality of $18 \%$ for patients who had an intermediate probability lung scan, very few of the deaths being attributable to PE."

A striking finding in this large outcome study is the observation that patients who had PE excluded (normal LSS) had no better long-term survival than those with PE confirmed (high probability LSS) when correcting for age and gender distribution. These data suggest that among patients with symptoms suggestive of $\mathrm{PE}$, thromboembolism per se may not be the principal prognostic determinant.
It is also intriguing that patients with an indeterminate scan did significantly worse than those with a normal or high probability LSS. This could be explained by the fact that many underlying cardiopulmonary diseases can predispose to an indeterminate LSS, resulting in a higher proportion of co-morbidities in this group. This is supported by the observation that patients with an indeterminate LSS had the highest proportion of abnormal CXRs. When including the CXR result in the analysis, the survival difference between patients with indeterminate and high probability LSS was still significant, albeit less strongly.

The manner in which patients with indeterminate lung scans are managed may also be important, and we cannot exclude the possibility that untreated PE may have contributed to mortality in our indeterminate LSS group. Pulmonary embolism is present in $30 \%$ of patients with intermediate probability interpretations and in $14 \%$ with low probability interpretations, ${ }^{5}$ and a nondiagnostic lung scan should not be considered an endpoint in itself. On evaluation of a subset of patients with non-diagnostic lung scans, we found that no further investigations were performed in $55 \% .^{12}$ It is likely that some of these had undiagnosed and untreated PE, and that others without PE may have been inappropriately treated. In either case this might contribute to the poor prognosis in the indeterminate LSS group.

The observations presented here are broadly in keeping with prospective data from our own group, suggesting that patients with no evidence of PE at CTPA have a short-term prognosis no better than patients in whom CTPA confirms PE. ${ }^{3}$ The emerging picture suggests that patients with clinically suspected PE in whom the diagnosis is refuted represent a heterogeneous and illdefined group which collectively has a poor prognosis. We believe this trend may be under-recognised, emphasising the need for further investigation in symptomatic patients with an indeterminate LSS and suggesting that this particular cohort of patients deserves further study. The PIOPED study states that an indeterminate LSS should not be an endpoint and advises that additional investigation for PE should be performed in this group. ${ }^{5}$ These days, such additional studies will usually take the form of a CTPA. Our data suggest that co-morbidities already known about or demonstrated by these additional studies may contribute significantly to subsequent mortality.

A further implication of our findings relates to the importance of the CXR in stratifying risk for patients who have LSS for suspected PE. ${ }^{14}$ Computed tomography pulmonary angiography is now the primary investigative tool in patients with suspected PE, and CTPA is particularly recommended for patients with an abnormal $C X R^{14}$ or with an indeterminate lung scan. Table 3 suggests that the $\mathrm{CXR}$ result was more influential in assessing survival than 
the LSS result. However, some caveats must be kept in mind. The retrospective nature of this study precludes accurate delineation of the pathological abnormalities underlying the CXR changes. The CXR abnormalities of a subset of patients from the same database were characterised in an earlier paper. $^{14}$ Individual CXR abnormalities (consolidation, pleural effusion, cardiomegally, chronic obstructive pulmonary disease, left ventricular failure, scarring and linear atalectasis) were all shown to independently increase the probability of a non-diagnostic LSS compared with a normal CXR.'2 These abnormalities will often reflect the presence of co-existing cardio-pulmonary disease.

Our study has the strength of including comprehensive numbers of patients for whom key data (LSS result and outcome) were available. However, we recognise that this retrospective analysis has a number of limitations that could have influenced our results and their interpretation. In particular the inter- and intra-observer variation in LSS reporting is not defined. In addition, we cannot stratify the patient groups according to pre-test probability of $\mathrm{PE}$, and therefore cannot comment on how clinically 'appropriate' scan requests were for each group. Nor can we guarantee that co-morbidities likely to impact significantly on survival were evenly distributed among the groups studied. It might be anticipated that co-morbidities which predispose to PE (e.g. extrathoracic malignancy/cerebrovascular disease $)^{15}$ are likely to be over-represented in the 'high probability' group, in which the diagnosis of PE is most secure. However, there is also likely to be a lower threshold for investigating such patients for suspected PE simply because their increased risk of $P E$ is well recognised. This in turn will lead to more patients without PE being investigated. The co-morbidities present may result in an abnormal CXR with more matched $\mathrm{CXR} /$ perfusion abnormalities and more non-diagnostic LSSs.
We also acknowledge that the method used for obtaining mortality data has its limitations, as it would not detect a patient's death if he or she died after migrating out of Scotland. However, according to the 200I UK Annual Census, the rate of migration out of Scotland is less than $1 \%,{ }^{16}$ so the number of patients missed is likely to be negligible. A further limitation of the study is that we cannot accurately comment on causes of death. Data from death certificates were available, with PE recorded as the cause of death, in only $4 \%$ (approximately twothirds of these in the high probability LSS group). However, as death certificates are so notoriously inaccurate we could not depend on the reliability of these data.

Finally, it is important to recognise that our observation of an indeterminate LSS predicting a poorer prognosis than high probability LSS holds true for unselected patients with suspected PE. When only patients with a normal CXR were considered, this difference was no longer statistically significant. The importance of this caveat lies in the fact that currently, in many healthcare systems, LSS is reserved for those patients with a normal CXR.

In conclusion, indeterminate scintigraphy scans are associated with a poorer prognosis than normal or high probability LSS. Our initial hypothesis that survival is inversely proportional to the radiological probability of PE has therefore not been supported by our data. Co-morbidities in patients with indeterminate LSS may contribute significantly to mortality. An abnormal CXR at the time of referral for diagnostic evaluation of $P E$ is a strong indicator of a poor outcome, irrespective of whether PE is present, and has the advantage of offering some clinical clues as to the nature of any significant co-morbidity. Among patients presenting with suspected $P E$ the relative contribution of $P E$ to overall prognosis requires to be better characterised whether investigated by LSS or by CTPA.

\section{REFERENCES}

I Stein PD, Fowler SE, Goodman LR et al. Multidetector computed tomography for acute pulmonary embolism. N Engl J Med 2006; 354:23 17-27. doi:I0.1056/NEJMoa052367

2 Guilabert JP, Manzur DN, Tarrasa MJ et al. Can multislice CT alone rule out reliably pulmonary embolism? A prospective study. Eur J Radiol 2007; 62:220-6. doi:10.1016/j.ejrad.2006.II.032

3 O'Neill JM, Wright L, Murchison JT. Helical CTPA in the investigation of pulmonary embolism: a 6-year review. Clin Radiol 2004; 59:819-25. doi:10.1016/j.crad.2004.02.01 I

4 Gray HW, Neilly JB. The role and value of ventilation perfusion imaging in pulmonary embolism. In Oudkerk M, van Beek EJ, ten Cate JW, editors. Pulmonary embolism. Berlin: Blackwell Science; 1999. p. 84-123.

5 Saltzman HA, Alavi A, Greenspan RH et al.; the PIOPED Investigators. Value of the ventilation/perfusion scan in acute pulmonary embolism. Results of the Prospective Investigation of Pulmonary Embolism Diagnosis (PIOPED). JAMA 1990; 263:27539. doi:I0.100I/jama.263.20.2753
6 Miniati M, Pistolesi M, Marini C et al.Value of perfusion lung scan in the diagnosis of pulmonary embolism: results of the Prospective Investigative Study of Acute Pulmonary Embolism Diagnosis (PISA-PED). Am J Respir Crit Care Med 1996; 154:1387-93.

7 Anderson DR, Kahn SR, Rodger MA et al. Computed tomographic pulmonary angiography vs ventilation-perfusion lung scanning in patients with suspected pulmonary embolism. A randomized controlled trial.JAMA 2007; 298:2743-53. doi:I0.I00I/jama.298.23.2743

8 Akaike H. A new look at the statistical model identification. IEEE Trans Automat Contr 1976;AC-19:716-23.

9 Goldhaber SZ, Visani L, de Rosa $M$ et al. Acute pulmonary embolism: clinical outcomes in the International Cooperative Pulmonary Embolism Registry (ICOPER). Lancet 1999; 353:13869. doi:I0.I0I6/SOI40-6736(98)07534-5

10 Hvitfeldt Poulsen S, Noer I, Moller JE et al. Clinical outcome of patients with suspected pulmonary embolism. A follow-up study of 588 consecutive patients. J Intern Med 200I; 250:137-43. doi:I0.1046/j.I365-2796.200I.00866.x 
I J Jacobson AF, Patel N, Lewis DH. Clinical outcome of patients with intermediate probability lung scans during six-month follow-up. J Nucl Med 1997; 38:1593-6.

12 Murchison JT, Gavan DR, Reid JH. Clinical utilisation of the nondiagnostic lung scintigram. Clin Radiol 1997; 52:295-8. doi: I0.1016/ S0009-9260(97)80057-8

I3 Akram AR, Cowell GW, Logan LJA et al. Clinically suspected acute pulmonary embolism: a comparison of presentation, radiological features and outcome in patients with and without PE. QJM 2009; 102:407-14. doi:10.1093/qjmed/hcp04 I
I4 Forbes KPN, Reid JH, Murchison JT. Do preliminary chest X-ray findings define the optimum role of pulmonary scintigraphy in suspected pulmonary embolus? Clin Radiol 200I; 56:397-400. doi:10.1053/crad.2000.0670

15 Hyers TM. Venous thromboembolism. Am J Respir Crit Care Med 1999; 159:I-14.

I6 Fleming AD. Scotland's census 200 I: statistics on migration. Edinburgh: General Register Office for Scotland; 2005.

\section{TH ST ANDREW'S DAY FESTIVAL SYMPOSIUM FIVE DECADES OF MEDICAL PROGRESS}

\section{Thursday 2 - Friday 3 December 2010}

Venue: Royal College of Physicians of Edinburgh

This year, the Royal College of Physicians of Edinburgh is organising its 50th St Andrew's Day Symposium. To mark the anniversary of the College's annual flagship event, this year's symposium will be slightly different in nature.

The programme will cover eight different specialty areas, looking at key developments over the past 50 years but with the emphasis very much on current and emerging clinical management issues.

The specialty areas covered will be:

\section{Cardiovascular medicine \\ Genetics \\ Neurology \\ Renal medicine}

To view the full programme and to book online please visit http://events.rcpe.ac.uk or contact:

Eileen Strawn

Symposium Co-ordinator

Royal College of Physicians of

Edinburgh

9 Queen Street

Edinburgh EH2 IJQ

Tel: 01312473619

E-mail: e.strawn@rcpe.ac.uk

The first St Andrew's Day Symposium was on genetics and cell biology, and the College is delighted that Professor David Porteous will give the Ballantyne Lecture on 'Our genetic inheritance: a decade on from sequencing the human genome'. In addition, Baroness Ilora Finlay will give the Sir James Cameron Lecture on 'Palliative care - 2020 vision'. 